\title{
Erratum: Measurement of neutrino and antineutrino oscillations by the T2K experiment including a new additional sample of $\nu_{e}$ interactions at the far detector [Phys. Rev. D 96, 092006 (2017)]
}

\author{
K. Abe et al.
}

(The T2K Collaboration)

(Received 16 July 2018; published 31 July 2018)

DOI: $10.1103 /$ PhysRevD.98.019902

After publication we realized that the results for the atmospheric mass squared difference $\left|\Delta m^{2}\right|$ were wrongly reported in the paper. The likelihood function in the Frequentist analysis is parametrized as a function of $\Delta m_{32}^{2}$ (where $m_{3}$ is the largest mass) for normal ordering and $\Delta m_{13}^{2}$ (where $m_{3}$ is the smallest mass) for inverted ordering. In the Abstract, Conclusions, Sec. XI. A and Table XXV, this was incorrectly reported as $\Delta m_{32}^{2}$ for both mass orderings.

The central values and $68 \%$ confidence intervals found in this analysis are $\Delta m_{32}^{2}=(2.54 \pm 0.08) \times 10^{-3} \mathrm{eV}^{2} / c^{4}$ for normal ordering and $\Delta m_{13}^{2}=(2.51 \pm 0.08) \times 10^{-3} \mathrm{eV}^{2} / c^{4}$ for inverted ordering. The correct version of the Table XXV is reported here in Table I. This mislabeling does not affect the best-fit and credible intervals for the Bayesian analysis described in Sec. XI. B and in Tables XXVII and XXIX of the paper.

TABLE I. Best-fit and $1 \sigma$ confidence interval of the T2K data fit with the reactor constraint with normal $\left(\Delta m_{32}^{2}\right)$ and inverted $\left(\Delta m_{13}^{2}\right)$ ordering hypotheses.

\begin{tabular}{lccccc}
\hline \hline & \multicolumn{2}{c}{ Normal ordering } & \multicolumn{2}{c}{ Inverted ordering } \\
\cline { 2 - 6 } Parameter & Best-fit & $\pm 1 \sigma$ & \multicolumn{2}{c}{ Best-fit } & {$[-2.170 ;-0.768]$} \\
\hline$\delta_{C P}$ & -1.728 & {$[-2.538 ;-0.877]$} & -1.445 & {$[0.470 ; 0.601]$} \\
$\sin ^{2} \theta_{23}$ & 0.550 & {$[0.465 ; 0.601]$} & {$[2.460 ; 2.621]$} & $\ldots$ & $\ldots$ \\
$\Delta m_{32}^{2}\left(10^{-3} \mathrm{eV}^{2} / c^{4}\right)$ & 2.54 & $\ldots$ & 2.51 & {$[2.429 ; 2.588]$} \\
$\Delta m_{13}^{2}\left(10^{-3} \mathrm{eV}^{2} / c^{4}\right)$ & $\ldots$ & $\ldots$ & $\ldots 25$ & $\ldots$ \\
\hline \hline
\end{tabular}

Published by the American Physical Society under the terms of the Creative Commons Attribution 4.0 International license. Further distribution of this work must maintain attribution to the author(s) and the published articles title, journal citation, and DOI. 